\title{
A CHARACTERIZATION OF STRONG REGULARITY OF INTERVAL MATRICES*
}

\author{
JIRI ROHN ${ }^{\dagger}$
}

Abstract. As the main result of this paper it is proved that an interval matrix $\left[A_{c}-\Delta, A_{c}+\Delta\right]$ is strongly regular if and only if the matrix inequality $M\left(I-\left|I-R A_{c}\right|-|R| \Delta\right) \geq I$ has a solution, where $M$ and $R$ are real square matrices and $M$ is nonnegative. Several consequences of this result are drawn.

Key words. Interval matrix, Strong regularity, Spectral radius, Matrix inequality, Solvability.

AMS subject classifications. 65G40.

1. Introduction. In this paper, we use the following notations: $I$ is the unit matrix, $\varrho(A)$ denotes the spectral radius of $A$, for $A=\left(a_{i j}\right)$ we denote its absolute value by $|A|=\left(\left|a_{i j}\right|\right)$, and matrix inequalities are understood componentwise.

An $n \times n$ interval matrix

$$
\mathbf{A}=\left[A_{c}-\Delta, A_{c}+\Delta\right]=\left\{A|| A-A_{c} \mid \leq \Delta\right\}
$$

is called strongly regular [3] if $A_{c}$ is nonsingular and

$$
\varrho\left(\left|A_{c}^{-1}\right| \Delta\right)<1
$$

holds. In view of well-known properties of nonnegative matrices (see the equivalence of (i) and (ii) in Theorem 2.1 below), the condition (1.1) can be equivalently written as

$$
\left(I-\left|A_{c}^{-1}\right| \Delta\right)^{-1} \geq 0 .
$$

Evaluation of the left-hand side of (1.2) requires computation of two exact inverses. In this paper, we show that the condition (1.2) can be equivalently replaced by the condition of solvability of the matrix inequality

$$
M\left(I-\left|I-R A_{c}\right|-|R| \Delta\right) \geq I,
$$

* Received by the editors on November 24, 2009. Accepted for publication on November 5, 2010. Handling Editor: Angelika Bunse-Gerstner.

${ }^{\dagger}$ Institute of Computer Science, Czech Academy of Sciences, Prague, and School of Business Administration, Anglo-American University, Prague, Czech Republic (rohn@cs.cas.cz). This work was supported by the Czech Republic Grant Agency under grants 201/09/1957 and 201/08/J020, and by the Institutional Research Plan AV0Z10300504. 
where $M, R \in \mathbb{R}^{n \times n}$ and $M$ is required to be nonnegative. In the preliminary announcement [6] from 1994, which was never followed by a full paper, this author mentioned that "it can be shown that matrices $R$ and $M \geq 0$ satisfying (1.3) exist if and only if (1.1) holds". Now, almost exactly 16 years after writing [6], the author has returned to the task of filling in this gap. This is done in Theorem 3.1 below whose consequence (Theorem 3.2) also states that if the inequality (1.3) has a solution $M \geq 0$ and $R$, then it also has a particular solution $M_{0}=\left(I-\left|A_{c}^{-1}\right| \Delta\right)^{-1} \geq 0$ and $R_{0}=A_{c}^{-1}$. Thus, $M$ and $R$ can be viewed as approximations of the exact inverses $M_{0}$ and $R_{0}$. In the last Theorem 4.1 we sum up some consequences of solvability of (1.3). In particular, we prove that if $M \geq 0, R$ solve (1.3), then

$$
\left|A^{-1}-R\right| \leq(M-I)|R|
$$

holds for each $A \in \mathbf{A}$. We do not give any applications of these results here, having in mind writing another paper about these issues.

2. Auxiliary results. In the proofs to follow, we shall essentially use spectral properties of nonnegative matrices that are summed up in the following two theorems. The proofs can be found in Horn and Johnson [4] or Meyer [5].

THEOREM 2.1. For a nonnegative square matrix $G$, the following assertions are equivalent:

(i) $\varrho(G)<1$,

(ii) $I-G$ is nonsingular and $(I-G)^{-1} \geq 0$,

(iii) $G^{j} \rightarrow 0$ as $j \rightarrow \infty$,

(iv) $G x<x$ for some $x>0$.

Moreover, if any of these conditions is met, then $(I-G)^{-1}=\sum_{j=0}^{\infty} G^{j}$.

Theorem 2.2. If $A, B \in \mathbb{R}^{n \times n}$ satisfy $|A| \leq B$, then $\varrho(A) \leq \varrho(|A|) \leq \varrho(B)$.

3. Characterization. In this section, we show that strong regularity of $\left[A_{c}-\right.$ $\left.\Delta, A_{c}+\Delta\right]$ is equivalent to solvability of the matrix inequality

$$
M\left(I-\left|I-R A_{c}\right|-|R| \Delta\right) \geq I,
$$

where $M, R \in \mathbb{R}^{n \times n}$, and $M$ is required to be nonnegative. Alternatively, we can also write $(3.1)$ as

$$
M(I-G) \geq I
$$

where

$$
G=\left|I-R A_{c}\right|+|R| \Delta
$$

is a nonnegative matrix. We have this result. 
Theorem 3.1. An interval matrix $\mathbf{A}=\left[A_{c}-\Delta, A_{c}+\Delta\right]$ is strongly regular if and only if the inequality (3.1) has a solution $M, R$, where $M \geq 0$.

Proof. Let (3.1) have a solution $M \geq 0$ and $R$. Then for $G$ given by (3.3), we have (3.2), which can be written as

$$
I+M G \leq M
$$

Postmultiplying this inequality by $G$ and adding $I$ to both sides we obtain

$$
I+G+M G^{2} \leq I+M G \leq M
$$

and by induction,

$$
\sum_{j=0}^{k} G^{j}+M G^{k+1} \leq M
$$

for $k=0,1,2, \ldots$ In view of nonnegativity of $M$, this shows that the nonnegative matrix series $\sum_{j=0}^{\infty} G^{j}$ satisfies

$$
\sum_{j=0}^{\infty} G^{j} \leq M
$$

hence it is convergent, so that $G^{j} \rightarrow 0$, and consequently

$$
\varrho(G)<1,
$$

by Theorem 2.1. Now we have

$$
I-R A_{c} \leq\left|I-R A_{c}\right| \leq G,
$$

and hence, by Theorem 2.2,

$$
\varrho\left(I-R A_{c}\right) \leq \varrho\left(\left|I-R A_{c}\right|\right) \leq \varrho(G)<1 .
$$

Since $\varrho\left(I-R A_{c}\right)<1$, the matrix

$$
R A_{c}=I-\left(I-R A_{c}\right)
$$

is nonsingular, which gives that both $A_{c}$ and $R$ are nonsingular. Moreover, (3.7) and Theorem 2.1 imply that

$$
A_{c}^{-1} R^{-1}=\left(R A_{c}\right)^{-1}=\sum_{j=0}^{\infty}\left(I-R A_{c}\right)^{j} .
$$


Hence,

$$
A_{c}^{-1}=\sum_{j=0}^{\infty}\left(I-R A_{c}\right)^{j} R
$$

and thus also

$$
\left|A_{c}^{-1}\right| \leq \sum_{j=0}^{\infty}\left|I-R A_{c}\right|^{j}|R|=\left(I-\left|I-R A_{c}\right|\right)^{-1}|R|
$$

(because $\sum_{j=0}^{\infty}\left|I-R A_{c}\right|^{j}$ is again convergent by (3.6)), and

$$
\left|A_{c}^{-1}\right| \Delta \leq\left(I-\left|I-R A_{c}\right|\right)^{-1}|R| \Delta .
$$

Since $\varrho(G)<1$, Theorem 2.1 implies existence of an $x>0$ satisfying $G x<x$, i.e.,

$$
\left|I-R A_{c}\right| x+|R| \Delta x<x,
$$

hence

$$
|R| \Delta x<\left(I-\left|I-R A_{c}\right|\right) x
$$

and

$$
\left(I-\left|I-R A_{c}\right|\right)^{-1}|R| \Delta x<x
$$

in view of (3.6). Now, from (3.8) and (3.9), we finally obtain

$$
\left|A_{c}^{-1}\right| \Delta x \leq\left(I-\left|I-R A_{c}\right|\right)^{-1}|R| \Delta x<x
$$

where $x>0$, hence $\varrho\left(\left|A_{c}^{-1}\right| \Delta\right)<1$ by Theorem 2.1, which proves that $\mathbf{A}$ is strongly regular. Conversely, if $\mathbf{A}$ is strongly regular, then $\left(I-\left|A_{c}^{-1}\right| \Delta\right)^{-1} \geq 0$ again by Theorem 2.1 and

$$
\begin{aligned}
M_{0} & =\left(I-\left|A_{c}^{-1}\right| \Delta\right)^{-1} \geq 0, \\
R_{0} & =A_{c}^{-1}
\end{aligned}
$$

satisfy (3.1) as an equation.

Thus, we have also proved the following result.

THEOREM 3.2. If the inequality (3.1) has a solution $M \geq 0$ and $R$, then it also has a particular solution $M_{0} \geq 0$ and $R_{0}$ given by (3.10) and (3.11). 
4. Consequences. Below, we list some consequences of solvability of the inequality (3.1).

TheOREM 4.1. Let (3.1) have a solution $M \geq 0$ and $R$. Then we have:

(a) $\varrho\left(\left|I-R A_{c}\right|+|R| \Delta\right)<1$,

(b) $\left(I-\left|I-R A_{c}\right|-|R| \Delta\right)^{-1} \leq M$,

(c) $\varrho\left(I-R A_{c}\right)<1$,

(d) $R$ is nonsingular,

(e) $\left[R^{-1}-\Delta, R^{-1}+\Delta\right]$ is strongly regular,

(f) each $A \in\left[A_{c}-\Delta, A_{c}+\Delta\right]$ is nonsingular and $A^{-1}=\sum_{j=0}^{\infty}(I-R A)^{j} R$,

(g) $\left|A^{-1}-R\right| \leq(M-I)|R|$ for each $A \in \mathbf{A}$.

Proof. The assertions (a), (c) and (d) have been proved in the proof of Theorem 3.1, and (b) is a consequence of (3.4). From (a), it follows that $\varrho(|R| \Delta) \leq \varrho(\mid I-$ $\left.R A_{c}|+| R \mid \Delta\right)<1$, which means that $\left[R^{-1}-\Delta, R^{-1}+\Delta\right]$ is strongly regular by definition, thus proving (e). To prove the remaining two assertions, take an $A \in \mathbf{A}$. Then it satisfies the identity

$$
R A=I-(I-R A)
$$

and since

$$
|I-R A|=\left|I-R A_{c}+R\left(A_{c}-A\right)\right| \leq\left|I-R A_{c}\right|+|R| \Delta=G,
$$

there holds

$$
\varrho(I-R A) \leq \varrho(G)<1
$$

by Theorem 2.2 and (3.5), so that (4.1) shows that $R A$ is nonsingular, hence $A$ is nonsingular. Next, from (4.1), it follows

$$
A^{-1} R^{-1}=\sum_{j=0}^{\infty}(I-R A)^{j}
$$

hence

$$
A^{-1}=\sum_{j=0}^{\infty}(I-R A)^{j} R
$$

which proves (f). Finally, from (4.3), (4.2) and (3.4), we have that

$$
\left|A^{-1}-R\right| \leq \sum_{j=1}^{\infty}\left(\left|I-R A_{c}\right|+|R| \Delta\right)^{j}|R|=\left(\sum_{j=0}^{\infty} G^{j}-I\right)|R| \leq(M-I)|R|,
$$

which concludes the proof. $\square$ 
We conclude this note by describing how the results presented here can be used to gather information about the inverse interval matrix. A square interval matrix $\mathbf{A}$ is called regular if each $A \in \mathbf{A}$ is nonsingular; a strongly regular interval matrix is regular (see Beeck [1]). For a regular interval matrix $\mathbf{A}$, its inverse interval matrix $\mathbf{A}^{-1}$ is defined as the narrowest interval matrix containing the set $\left\{A^{-1} \mid A \in \mathbf{A}\right\}$. Computing the inverse interval matrix is NP-hard (see Coxson [2]). Therefore, in practical computations we usually resort to enclosures of the inverse, i.e., to interval matrices containing $\mathbf{A}^{-1}$, but not necessarily the minimal ones with respect to inclusion. The assertion (g) of Theorem 4.1 says that if $M \geq 0$ and $R$ solve (3.1), then

$$
[R-(M-I)|R|, R+(M-I)|R|]
$$

is an enclosure of $\left[A_{c}-\Delta, A_{c}+\Delta\right]^{-1}$.

Acknowledgment. The author wishes to thank the referee for comments that resulted in improvement of the text of this paper.

\section{REFERENCES}

[1] H. Beeck. Zur Problematik der Hüllenbestimmung von Intervallgleichungssystemen. In K. Nickel, editor, Interval Mathematics, Lecture Notes in Computer Science, Springer-Verlag, Berlin, 29:150-159, 1975.

[2] G.E. Coxson. Computing exact bounds on elements of an inverse interval matrix is NP-hard. Reliable Computing, 5:137-142, 1999.

[3] M. Fiedler, J. Nedoma, J. Ramík, J. Rohn, and K. Zimmermann. Linear Optimization Problems with Inexact Data. Springer-Verlag, New York, 2006.

[4] R.A. Horn and C.R. Johnson. Matrix Analysis. Cambridge University Press, Cambridge, 1985.

[5] C.D. Meyer. Matrix Analysis and Applied Linear Algebra. SIAM, Philadelphia, 2000.

[6] J. Rohn. A perturbation theorem for linear equations. Commentationes Mathematicae Universitatis Carolinae, 35:213-214, 1994. 